\title{
Deshabituación de drogas y funcionamiento cerebral: una visión integradora
}

\author{
iñaki Lorea Conde*; Javier Tirapu Ustárroz**; Natalia Landa***; José Javier López-Goñi* \\ * Fundación Proyecto Hombre Navarra \\ * Servicio de Neuropsicología. Clínica Ubarmin. Elcano (Navarra) \\ *** Centro de Salud Mental de Tudela (Navarra). \\ Enviar correspondencia a: \\ Iñaki Lorea Conde. Centro de Día ALDATU. Fundación Proyecto Hombre Navarra. Avda. Zaragoza 23. 31005. Pamplona. \\ E-mail: centrodedia@proyectohombrenavarra.org
}

Recibido: 15 de enero de 2004

Aceptado: 17 de febrero de 2005.

\section{RESUMEN}

En la actualidad todavía estamos lejos de contar con modelos de comprensión y tratamiento de las adicciones que se sustenten en el funcionamiento cerebral. El objetivo de este trabajo es elaborar un modelo integrador entre psicología clínica y neuropsicología con el fin de anclar nuestras intervenciones clínicas en enfoques que nos acerquen al discurso de la neurociencia. Más en concreto, se revisan tres modelos de intervención de amplia utilización en adicciones como son la entrevista motivacional, la prevención de recaídas y el trabajo con redes sociales. Posteriormente, se relacionan estos enfoques con su posible efecto en el funcionamiento cerebral, basándonos en el reconocido "modelo jerárquico" de Stuss y Benson y en el modelo de circuitos cerebrales propuesto por Volkow, Fowler y Wang. Así cada intervención tendría como objetivo eliminar diversas funciones cerebrales que se ven modificadas durante el proceso adictivo. Las intervenciones propias de la entrevista motivacional mejorarían el funcionamiento del nivel superior de procesamiento cerebral, aumentando la conciencia del problema. Estrategias del trabajo con redes sociales como la detección de situaciones de riesgo o la evitación de estímulos condicionados incidirían en los niveles de procesamiento básico y de control de activación de acciones. Técnicas propias de la prevención de recaídas como la exposición con prevención de respuesta o el manejo del E.V.A. influirían en el funcionamiento de los niveles de autocontrol y de funciones básicas. Además, estos enfoques de tratamiento también modificarían los circuitos de recompensa, motivación, memoria y control cognitivo. En definitiva, la finalidad es someter al sujeto una serie de experiencias para que las relaciones cerebro-mente se orienten en una dirección más adaptativa.

Palabras clave: Adicción, Entrevista Motivacional, Modelo Jerárquico, Neurociencia, Prevención de Recaídas, Redes Sociales.

\section{ABSTRACT}

We are still a long way away from having appropriate models to understand and treat addictions sustained by brain functions. The aim of this paper is to develop a model integrating psychology and neuropsychology in order to refocus our clinical interventions closer to the neuroscience discourse. Specifically, three widely used treatment models for addictions are reviewed here, Motivational Interview, Relapse Prevention and Work with Social Networks. Subsequently, these different approaches will be linked to their possible effect on the cerebral functions, based on the well-known Hierarchical Model (Stuss and Benson) as well as the Brain Circuits Model (Volkow, Fowler and Wang). Therefore, each intervention should target several brain functions modified during the addiction process. The motivational interview should improve the functioning of the higher level of the brain processing, increasing the awareness of the problem. Strategies with the social networks such as the detection of high-risk situations or the avoidance of conditioned stimuli should have an impact on both the basic processing and the action activation control levels. Relapse prevention techniques such as exposure with response prevention, or E.V.A. handling, should influence the working of the levels of selfcontrol and of basic functions. Furthermore, these treatment approaches should also change the reward, motivation, memory and cognitive control circuits. In conclusion, the objective is to make the individual undergo a set of experiences in order to re-orient the brain-mind relationship towards a more adaptive direction.

Key words: Addiction, Motivational Interview, Hierarchical Model, Neuroscience, Relapse Prevention, Social Networks.

\section{INTRODUCCIÓN}

na de las características más peculiares de la psicología y la psiquiatría es la variedad de marcos conceptuales de explicación de la psi- copatología. Ante el mismo fenómeno psicopatológico, cada modelo propone su explicación y tratamiento: la psiquiatría biológica se refiere a desequilibrios de neurotransmisores, la psicología cognitiva a distorsiones en el estilo de pensamiento y de la construcción 
de la realidad interna y el psicoanálisis indagará en las experiencias tempranas que condicionan procesos inconscientes.

Este panorama tan diverso es resultado de una doble dificultad: en primer lugar, la de encontrar un marco común de comprensión de las enfermedades mentales y en segundo lugar, la dificultad derivada de la ausencia de un paradigma que proporcione una explicación al origen y funcionamiento de la mente nor$\mathrm{mal}^{(1)}$.

En el ámbito de los trastornos adictivos, el panorama es similar. El abordaje histórico de las drogodependencias ha estado muy influido en sus inicios por modelos moralistas que consideraban este desorden como un problema de índole moral más que una enfermedad ${ }^{(2)}$. En la actualidad, aunque aún se conservan resquicios de estas concepciones pasadas, las disciplinas que estudian las alteraciones cerebrales que ocurren durante los procesos adictivos se están abriendo paso con fuerza. Disciplinas como la neurobiología o técnicas como la neuroimagen cerebral están proporcionando un importante caudal de información sobre fenómenos cerebrales asociados a la adicción, aunque adolecen de una teoría cerebro-mente sólida en la que integrar sus resultados.

El desarrollo del conocimiento neurocientífico nos ha enseñado que durante la instauración del proceso adictivo, el cerebro humano adquiere una neuroadaptación bioquímica y neuronal. Asimismo, es muy probable que el cerebro humano adquiera paralelamente una neuroadaptación funcional, es decir una neuroadaptación en la dinámica integradora de sus funciones cognitivas y ejecutivas o volitivas ${ }^{(5)}$. Estas funciones tienen que ver con la capacidad de atención, concentración, integración, procesamiento de la información, y ejecución de planes de acción consecuentes con dicha información.

Como se ha señalado recientemente(3) ${ }^{(3)}$ cuando intentamos describir y profundizar en un comportamiento humano como la adicción partimos de un marco conceptual determinado. Kandel(4) en un magnífico artículo titulado "Hacia un nuevo marco conceptual en psiquiatría" aborda este tema y sus conclusiones aplicadas a las adicciones pudieran ser como siguen: 1) los procesos mentales derivan de las operaciones del cerebro por lo que la adicción sería una alteración de la función cerebral, 2) los genes señalan un patrón de interconexión neuronal por lo que ejercen un control significativo sobre la conducta, la adicción tiene un componente genético, 3) los genes alterados no pueden explicar la adicción, la conducta social ejerce acciones sobre el cerebro retroalimentándolo para modificar la expresión de los genes y, por consiguiente, la función de las neuronas (lo educativo es expresado como "natural o biológico"), 4) existe un alteración en el patrón de manifestación de los genes inducida por el aprendizaje y contingencias sociales y 5) la terapia es eficaz sólo si produce cambios a largo plazo en la conducta, porque entonces la conducta produce cambios en la expresión de los genes que alteran el patrón anatómico de las interacciones entre las células nerviosas del cerebro.

Este último aspecto adquiere una gran relevancia por ser el que menos se ha tenido en cuenta a la hora de establecer las relaciones entre cerebro y conducta. ¿Cómo afecta la intervención terapéutica a las conexiones neurales? Una premisa fundamental en las relaciones cerebro-mente es que cualquier proceso mental y cualquier conducta responden a un patrón de conexiones entre neuronas. Por lo tanto, estaremos de acuerdo en afirmar que una intervención será eficaz en tanto en cuanto sea capaz de modificar las pautas de conexión interneuronal. De hecho, consideramos que en el futuro uno de los pilares básicos para sustentar la "medicina basada en la evidencia" o la intervención terapéutica basada en la evidencia van a ser las técnicas de neuroimagen con las que se valorará cómo una intervención ha modificado el funcionamiento cerebral.

El objetivo de este trabajo es elaborar un modelo integrador de acercamiento entre la psicología clínica y la neuropsicología con el fin de establecer modelos que permitan anclar nuestras intervenciones clínicas en el funcionamiento cerebral. Pretendemos, pues, tender puentes entre niveles de análisis que nos conduzcan hacia la ciencia analítica y nos provean de modelos hipotéticos para acercarnos al discurso de la neurociencia.

\section{MODELOS DE DESHABITUACIÓN EN DROGO- DEPENDENCIAS: ENTREVISTA MOTIVACIONAL, PREVENCIÓN DE RECAÍDAS, REDES SOCIA- LES.}

\subsection{La Entrevista Motivacional.}

El tratamiento de cualquier adicción se ha topado muchas veces con la dificultad de un paciente "sin conciencia del problema", "poco motivado" o "carente de voluntad de cambio". Estos problemas suelen representar objetivos fundamentales de cualquier intervención terapéutica. En este sentido, el modelo de Entrevista Motivacional desarrollado por Miller y Rollnick en $1991^{(6)}$ representa un estilo de asistencia directa, centrada en el paciente y que trata de provocar un cambio en su comportamiento, a través de ayudarle a explorar y resolver ambivalencias. Podríamos definir la ambivalencia como la presencia de emociones contrapuestas con respecto al abandono del consumo 
de sustancias, algo así como "quiero dejar de tener problemas por consumir cocaína, pero sin dejar de consumir cocaína".

Este modelo de intervención es más un estilo de relación terapéutica que un modelo de tratamiento en particular, por lo cual, permite su ejecución de manera integrada con otros modelos de deshabituación de drogas (como el de Prevención de Recaídas, por ejemplo) y en cualquier fase de la intervención (desintoxicación-deshabituación-mantenimiento).

La investigación reciente en pacientes adictos a drogas señala que el enfoque de tratamiento basado en Entrevista Motivacional durante el proceso de evaluación aumenta el compromiso de tratamiento posterior $^{(7)}$. Asimismo, mejora la retención en tratamientos ambulatorios para pacientes psiquiátricos y con patología dual después de un ingreso hospitalario(8). Stotts, Schmitz, Rhoades y Grabowski, ${ }^{(9)}$ comparan un programa de desintoxicación ambulatorio para adictos a la cocaína con y sin la aplicación de Entrevista Motivacional. Sus resultados indican que la práctica de Entrevista Motivacional mejora el compromiso de utilización de estrategias de afrontamiento, aunque no obtuvieron diferencias significativas respecto a la retención en tratamiento.

\subsection{El modelo de Prevención de Recaídas.}

Uno de los modelos de tratamiento más ampliamente aceptados en el ámbito de las drogodependencias es el de prevención de recaídas propuesto por Marlatt y Gordon en 1985(10). Se trata de un programa de autocontrol diseñado con el fin de ayudar a los pacientes a anticipar y afrontar los problemas de la recaída en el cambio de las conductas adictivas. Combina procedimientos conductuales de entrenamiento en habilidades, terapia cognitiva y el reequilibrio del estilo de vida. Algunos aspectos clave de este enfoque giran alrededor de la diferenciación entre caída y recaída, la detección de las denominadas situaciones de alto riesgo, el "efecto de la violación de la abstinencia", la "expectativa de autoeficacia", las "decisiones aparentemente irrelevantes" y las "estrategias de afrontamiento".

De manera resumida, este programa facilita que el paciente conozca cuáles son los factores relacionados con sus dificultades para lograr un estado de abstinencia y le ayuda a afrontar los problemas que se encontrará en una vida sin drogas. En esta labor, un aspecto fundamental lo constituye la forma en que el sujeto detecta y afronta las situaciones de alto riesgo. En conjunto y con un carácter marcadamente cognitivo, aporta una explicación del fenómeno muy comprensible, junto con herramientas de intervención concretas y sencillas (habilidades sociales, relajación, control de la ira) y es indiscutible que aporta claridad a un fenómeno tan confuso como el de la adicción a drogas.

\subsection{El trabajo con redes sociales.}

Por su parte, el elemento ambiental también contribuye al desarrollo del trastorno adictivo y desempeña un papel fundamental en el proceso de rehabilitación. La investigación se ha centrado principalmente en la función que algunos elementos ambientales como el estrés y los estímulos condicionados desempeñan en el proceso adictivo(11).

En este sentido, conceptos como red social, apoyo social o apoyo familiar focalizan la atención hacia el elemento ambiental y su influencia en la deshabituación ${ }^{(12,13)}$. Además, nos proporcionan estrategias de análisis e intervención a fin de controlar el efecto del estrés y los estímulos condicionados. Por ejemplo, el hecho de disponer de una red que brinde apoyo social (como la familia) no presupone un efecto positivo, ya que bajo ciertas circunstancias, la red social puede convertirse en fuente de estrés. Entonces, la presencia de conflictos de relación dentro de la familia puede actuar como un estresor ambiental que facilite la ocurrencia de una recaída. En segundo lugar, la presencia de compañeros consumidores o la frecuentación de entornos en los que se ha consumido drogas, actúan como estímulos condicionados que facilitarán la respuesta de consumo de drogas.

Desde este punto de vista, el trabajo sobre la red social irá orientado hacia: a) minimizar el apoyo de la red al consumo de drogas, b) vincular a la red nuevos sujetos con estilos de vida libres de drogas y c) fomentar la estima del adicto hacia los miembros de su red.

Aunque existe consenso sobre la importancia de las variables ambientales en la evolución de los trastornos adictivos en general, se necesitan más estudios controlados que nos ayuden a comprender mejor el peso de estas variables. Cabe destacar el trabajo de Broome, Simpson y Joe ${ }^{(14)}$ sobre una muestra de 748 pacientes con trastornos por uso de alcohol y cocaína, en el que concluyen que la red de apoyo social de los pacientes que continúan en tratamiento es un factor más importante que otras medidas pretratamiento. Es decir, constataron que contar con dicha red (o contar con una red adecuada) mejora los resultados de los programas de intervención breve respecto al uso de cocaína y alcohol en el seguimiento. También se ha relacionado el bajo apoyo social con el aumento de posibilidades de abandonar prematuramente un tratamiento de deshabituación ambulatorio(15), aunque el nivel de apoyo social demostró ser un modesto predictor de la severidad de abuso de alcohol. Asimismo, se han 
encontrado asociaciones entre el nivel de apoyo social en la admisión en un programa de mantenimiento con metadona o LAAM con la abstinencia de heroína y cocaína a los 3 meses de tratamiento(16).

\section{MODELOS DE FUNCIONAMIENTO CEREBRAL}

\subsection{El modelo jerárquico de Stuss y Benson.}

Uno de los fenómenos que con más frecuencia aparece en los trastornos adictivos y que dificulta su abordaje es la falta de conciencia del problema. Esta constatación nos obliga a reflexionar sobre qué es la conciencia y a buscar referentes teóricos en este ámbito.

Los trabajos sobre los mecanismos de la conciencia han coincidido en señalar la afinidad de los lóbulos frontales por este tipo de funciones y entre los estudios sobre la conciencia, destaca el modelo de jerarquización de las funciones mentales de Stuss y Benson, propuesto en 1986 en su libro sobre el lóbulo frontal ${ }^{(17)}$. Como puede observarse en la figura 1, las funciones mentales básicas como atención, lenguaje, memoria, etc., están en el nivel más elemental de funcionamiento mental y se encuentran integradas entre sí, de tal manera que la activación de una de ellas significa la activación de otras y por lo tanto, la implicación de diferentes regiones corticales y subcorticales de ambos hemisferios. Estas funciones tendrían afinidad por regiones cerebrales basales y retrorrolándicas.

En un nivel superior, se encuentran las funciones de secuenciación e integración de la información, así como del impulso o motivación para la acción. Están relacionadas de manera interactiva con las de $4^{\circ}$ orden y muestran una mayor afinidad, aunque no de manera exclusiva, por regiones frontales: áreas prefrontales dorsolaterales regularían las funciones de secuenciación y regiones mediales las de impulso y motivación ${ }^{(18)}$. Estos circuitos regulan comportamientos rutinarios, hábitos de conducta que se realizan con frecuencia. Cuando una actividad se vuelve rutinaria, pasa a ser controlada por este nivel de control de activación de acciones.

En cambio, cuando un sujeto se expone a nuevos problemas o situaciones no rutinarias, pone en marcha una serie de estrategias cognitivas de alto nivel para responder de manera adecuada. En estas situaciones, el sujeto explora, monitoriza, selecciona, se concentra, cambia y evalúa toda la actividad del sistema nervioso. Estas funciones pueden resumirse en una función general de autocontrol o autorregulación de la conducta, funciones que están mediatizadas por regiones pre- frontales. Como señalan Tirapu, Muñoz-Céspedes y Pelegrín ${ }^{(19)}$, probablemente estas funciones emergen cuando el ser humano adquiere la capacidad cognitiva de la anticipación

Finalmente, en el vértice de la pirámide se encuentra la función de la autoconciencia, cuyo objetivo es el desarrollo de un conocimiento o conciencia individual que interactúa con el ambiente de una manera apropiada tanto a nivel individual como social. Además, representa las experiencias subjetivas actuales y las compara con las previas y controla la actividad mental en general. Esta tarea de orden superior estaría íntimamente relacionada con regiones prefrontales ${ }^{(18)}$.

La conducta es el resultado final de la actividad conjugada de estos 4 niveles (funciones básicas, control de activación de acciones, autocontrol y autoconciencia) y en función del nivel de rutina-novedad de la situación, la implicación de áreas prefrontales será menor o mayor.

\subsection{El modelo de circuitos cerebrales de Volkow, Fowler y Wang.}

Los resultados de la investigación con técnicas de neuroimagen cerebral ponen en evidencia que un amplio abanico de circuitos neuronales se ven afectados por el abuso de drogas. Esto es importante porque identificar y comprender los mecanismos que disregulan el funcionamiento de determinadas regiones cerebrales durante la instauración del trastorno adictivo permitiría actuar terapéuticamente sobre dichos procesos y sistemas. Entre estos estudios, destaca el trabajo de Volkow, Fowler y Wang, ${ }^{(20)}$, que han propuesto un modelo, basado en hallazgos de neuroimagenl, sobre los circuitos cerebrales implicados en el refuerzo, la motivación, la memoria y el control cognitivo y que ven modificado su funcionalismo con el abuso de drogas (Figura 2).

El circuito de recompensa lo componen núcleos cerebrales como el accumbens y el pálido ventral. La administración de drogas provoca un aumento de los niveles de dopamina en estos centros, incremento que está asociado a los efectos reforzantes de la sustancia. El consumo de drogas provoca un aumento de dopamina en el accumbens que son de 3 a 5 veces mayor que el ocasionado por los estímulos naturales (comida, por ejemplo). Posteriormente, la repetición de las autoadministraciones de drogas provocaría un proceso de neuroadaptación en forma de disminución de receptores DA D2, que insensibilizaría al sujeto hacia el refuerzo proveniente de estímulos naturales y le predispondría hacia las recaídas.

Por su parte, unas regiones fundamentales del circuito de motivación son el córtex órbitofrontal y el 


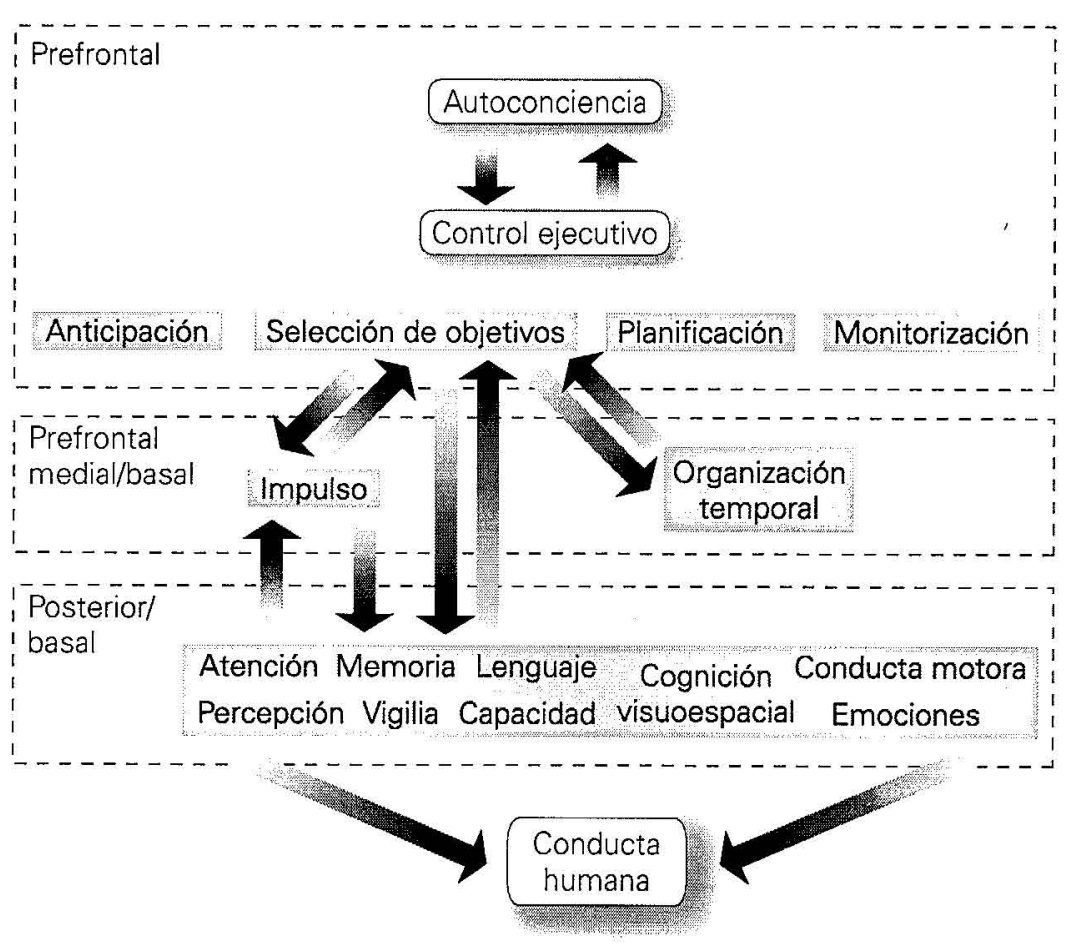

Figura 1. Modelo jerárquico de Stuss y Benson.
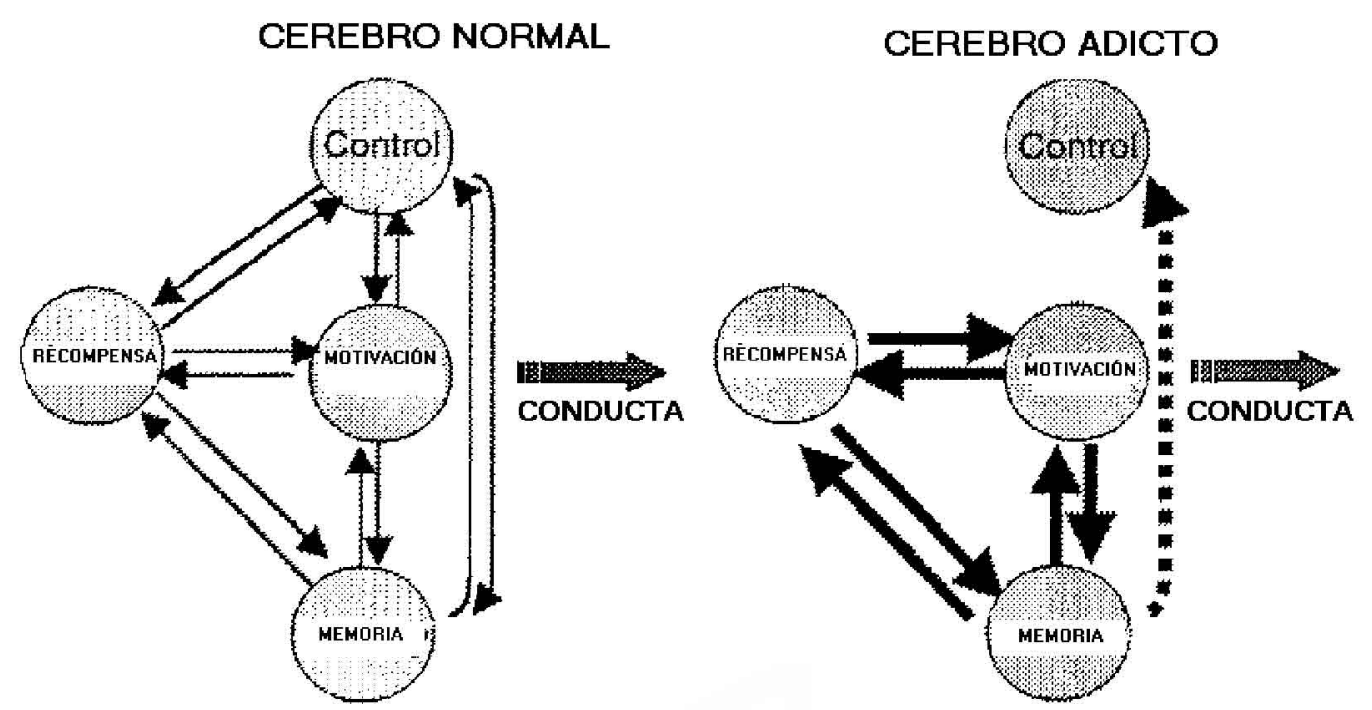

Figura 2. Circuitos cerebrales implicados en la adicción a drogas según Volkow, Fowler y Wang.

córtex subcalloso, áreas que procesan información asociada con la predicción de la recompensa y que regulan el valor asignado a los estímulos naturales en función de las necesidades internas del sujeto. Por ejemplo, el valor motivacional de la comida está en relación al estado interno del organismo, según se tenga hambre o no. Por el contrario, durante la instauración del trastorno adictivo, el valor motivacional de la droga es mayor que el de cualquier otro reforzador natural y además se vuelve fijo, deja de estar en función de las necesidades del organismo.

Varios sistemas de aprendizaje y memoria se encuentran afectados por la adicción a drogas: núcleos que regulan el condicionamiento clásico (amígdala, núcleo accumbens), el aprendizaje de hábitos (caudado y putamen) y la memoria declarativa (hipocampo) se hallan alterados en la adicción y pueden favorecer 
que el consumo de drogas se desencadene automáticamente a partir de la exposición a señales asociadas a drogas.

Finalmente, también se han hallado anormalidades en sujetos adictos sobre el funcionamiento de regiones cerebrales que regulan la toma de decisiones y la inhibición conductual, como el córtex prefrontal y el giro cingulado anterior. Estas disregulaciones provocan un funcionamiento erróneo en la toma de decisiones, al primar la recompensa inmediata sobre otras conductas que proveen de refuerzo diferido, aunque aquellas sean más favorables en términos de supervivencia. Así, la disminución del autocontrol conductual, regulado por áreas frontales, dejaría a la conducta en manos de otros centros subcorticales que son más sensibles a la recompensa inmediata (como la amígadala, por ejemplo).

\section{DESCRIPCIÓN DE INTERACCIONES ENTRE AM- BOS MODELOS}

La terapia de deshabituación que abordan los enfoques descritos con anterioridad trata de incidir en el funcionamiento mental del paciente para hacer que éste recupere el control sobre el consumo de sustancias.

Estos modelos de intervención provocan cambios que afectan a todos niveles jerárquicos del modelo de Stuss y Benson (tabla 1): se pretende mejorar la conciencia del problema (nivel de autoconciencia), el autocontrol (nivel de control ejecutivo), mejorar la motivación y reducir el "craving" (nivel de control de activación de acciones) y finalmente, modificar las percepciones, aminorar el significado emocional de la sustancia y desconectar las asociaciones entre estímulos condicionados e incondicionados (nivel de funciones mentales básicas).

A continuación, vamos a intentar describir qué tipo de intervenciones afectarían a cada nivel y qué modificaciones produciría cada intervención dentro del sistema de funciones mentales. Todos los enfoques de tratamiento se organizan dentro del mismo marco de intervención, aunque la intensidad de utilización de cada uno pueda cambiar a lo largo del tiempo.

El enfoque de la relación terapéutica basada en los elementos de la entrevista motivacional se mantendría más bien constante a lo largo de todo el tratamiento. Las intervenciones dirigidas a aumentar la conciencia del problema y a reforzar la voluntad de cambio se encuadrarían dentro del primer nivel de procesamiento supramodal (autoconciencia) y la estimulación efectiva de estas regiones tendría un efecto "liberador" sobre niveles de procesamiento inferior, facilitando la motivación hacia la puesta en marcha de nuevas estrategias conductuales, que a su vez movilizarían funciones cognitivas básicas (focalización de la atención en otros contextos ambientales...). El trabajo clínico cotidiano refleja este fenómeno en forma de un mayor porcentaje de asistencia a las consultas, un mejor cumplimiento de tareas (autorregistros...) y una mayor disposición hacia la realización de cambios conductuales indicados en la terapia.

\begin{tabular}{|llll|}
\hline \multicolumn{4}{|c|}{ Tabla 1. Cuadro-resumen de integración de modelos de intervención y funciones mentales } \\
(modelo jerárquico).
\end{tabular}


Conseguir y mantener un adecuado nivel de conciencia y motivación al cambio es un elemento fundamental a lo largo del tratamiento, así que este tipo de intervención mantendrá un alto grado de intensidad en toda nuestra actuación.

Posteriormente, una adecuada conciencia del problema nos permitiría comenzar a trabajar con elementos propios de las redes sociales y de la prevención de recaídas, como la detección de "situaciones de alto riesgo". Ésta es una tarea que se realiza en el ámbito de la consulta de evaluación y requiere que el paciente recuerde (función básica de memoria) en qué tipo de contextos, tanto externos (lugares, días de la semana...) como internos (estados emocionales o físicos) suele ser más frecuente que ocurran sus consumos de drogas.

Una vez detectadas las "situaciones de alto riesgo", el enfoque lo realizaremos a través de estrategias de evitación. De esta manera, tratamos de conseguir que el paciente alcance un período inicial de abstinencia a través de evitar que se exponga a percepciones $\left(4^{\circ} \mathrm{ni}-\right.$ vel) que están asociadas al consumo de drogas y que fruto de su emparejamiento contingente con la autoadministración de drogas, han adquirido la capacidad de desencadenar el deseo y las conductas necesarias (3 ${ }^{e r}$ nivel de impulso y secuenciación conductual) para consumir la sustancia.

Las estrategias iniciales de evitación se situarían en el nivel más básico y al prescindir de estímulos condicionados (detectados en la terapia), se impediría la realización de la secuencia de consumo. El resultado inicial de esta intervención sería una mejora sensible, aunque frágil, del autocontrol conductual (situado en $2^{\circ}$ nivel de jerarquía).

Posteriormente, se inicia el modelado de conductas de afrontamiento a través de técnicas de terapia de conducta como la exposición con prevención de respuesta. Durante la exposición a estímulos condicionados se ponen en juego funciones básicas de percepción y se organiza el contexto de tal manera (con la compañía de un familiar, por ejemplo) que se garantice la inhibición (función propia del $2^{\circ}$ nivel de autocontrol conductual) del impulso, que está regulado por las funciones de tercer nivel.

En estas situaciones se añaden algunos elementos de refuerzo de nivel básico previamente establecidos en la terapia, como el recuerdo de acontecimientos negativos asociados al consumo de drogas o de acontecimientos positivos asociados a la abstinencia (manejo de la memoria). Cuando la repetición de este esquema conductual es capaz de reducir el impulso, la consecuencia es una mejora más sólida en las funciones de autocontrol, ya que los procesos de inhibición conductual son capaces de efectuarse en contextos de mayor riesgo.
Cuando un sujeto evoluciona favorablemente en el tratamiento, múltiples cambios ocurren en los diversos niveles de su funcionamiento mental. Los procesos cognitivos básicos se hallan menos asociados a señales de drogas, la motivación hacia la autoadministración de la sustancia ha disminuido y el sujeto es más capaz de controlar su conducta, de elegir objetivos adecuados y de monitorizar el grado de consecución. Como resultado de esta acción conjunta, la propia conciencia del problema, en el nivel superior, cambia y el sujeto adquiere una mayor sensación de control, que el modelo de prevención de recaídas denomina "sensación de autoeficacia". Cuando todo el sistema trabaja en dirección adecuada, fuerza un cambio en la autoconciencia, que se traduce en que el sujeto aumenta su propia sensación de capacidad para afrontar de forma eficaz una situación de alto riesgo ("sé que puedo controlar la situación"). El efecto de estos cambios se expresa en la conducta, disminuyendo la probabilidad de que ocurran nuevas recaídas.

Sin embargo, se sabe de la frecuencia con que los fallos (entendidos como consumos puntuales) y/o las recaídas aparecen a lo largo del proceso de deshabituación. Estas dificultades, que aparecen a nivel de las funciones básicas y del control de activación de acciones, informarían a los niveles superiores de áreas o momentos de vulnerabilidad ante la conducta adictiva. La prevención de recaídas trata de controlar estos sucesos a través del aprendizaje de secuencias conductuales que habrán de ponerse en marcha lo antes posible tras el primer fallo, y que incluyen estrategias cognitivas y comportamentales (llamar inmediatamente al centro de tratamiento, por ejemplo). Estas estrategias se diseñan en la terapia y pretenden incluir un elemento de autocontrol situado en el $2^{\circ}$ nivel, que sea capaz de inhibir las secuencias de conductas sobreaprendidas de niveles inferiores.

Por su parte, Volkow, Fowler y Wang sugieren una serie de aproximaciones terapéuticas, tanto farmacológicas como conductuales y cognitivas, destinadas a revertir las disfunciones de cada uno de los sistemas neuronales afectados. A fin de disminuir la percepción de recompensa de las drogas, señalan la utilidad de abordajes farmacológicos que interrumpan el placer contingente a la conducta de autoadministración, o bien hagan que ésta tenga efectos desagradables. Los tratamientos con antagonistas o interdictores son algunos ejemplos de este tipo de intervenciones. La utilización de técnicas de evitación de estímulos, característica de la prevención de recaídas en etapas iniciales también modificaría el funcionalismo de este circuito, al permitir un período inicial de abstinencia que regule la presencia de receptores DA D2 hacia niveles premórbidos 21$)$.

Respecto al circuito de motivación, sugieren intervenciones que promuevan el valor de refuerzo de es- 
tímulos naturales alternativos al consumo de drogas, así como de conductas placenteras alternativas. La modificación del estilo de vida que proponen la prevención de recaídas y el trabajo con redes sociales iría orientado en este sentido, ya que encaminan al sujeto a exponerse a otra serie de conductas, hábitos de vida y actividades diarias que, al estar disociadas del consumo de drogas, aumentarán la motivación del sujeto por reforzadores alternativos. Dada la especial afinidad de estas regiones (córtex orbitofrontal, núcleo sub-calloso) por el procesamiento de información de índole emocional, parecería adecuado seleccionar las actividades alternativas en función de las características y preferencias individuales para garantizar que sean reforzantes para el sujeto y así tengan un mayor valor motivacional.

Respecto la las intervenciones dirigidas a revertir la disfunción de los sistemas de aprendizaje y memoria, sugieren diseñar intervenciones, como las técnicas conductuales de extinción, que debiliten la asociación entre el consumo de drogas y las señales que lo predicen. Las técnicas de exposición con prevención de respuesta propias de la prevención de recaídas tratan de incidir sobre estos circuitos y generar nuevos aprendizajes. Estos aprendizajes se traducirían en la desconexión de los circuitos implicados en la asociación de estímulos ambientales con la conducta de autoadministración de drogas.

Finalmente, proponen intervenciones de corte cognitivo para fortalecer el control inhibitorio en la toma de decisiones, regulado por circuitos frontales. Las intervenciones terapéuticas dirigidas a mejorar la conciencia del problema que utiliza la entrevista motivacional, como el empleo de técnicas de escucha activa a fin de seleccionar y encontrar las preocupaciones más significativas del paciente y de este modo provocar en él el deseo de cambio, serían un buen ejemplo de este tipo de acciones.

\section{CONCLUSIONES}

La neurociencia ha avanzado en los últimos años como no la había hecho en toda la historia de la humanidad. Estos nuevos hallazgos van a plantear serios interrogantes tanto a los modelos sobre la etiología de los trastornos como a los modelos de intervención. Ya no es creíble hacer afirmaciones basadas en "mi experiencia" e incluso se cuestionan algunas afirmaciones basadas en "no siempre evidentes pruebas basadas en la evidencia científica".

Una manera (no excluyente) de solventar esta difícil disyuntiva en la que nos encontramos, se basa en intentar establecer modelos de integración entre la neuropsicología y la psicología clínica ya que aquella disciplina debería forzar a las técnicas psicoterapéuticas a demostrar su eficacia evaluando los cambios sobre el funcionamiento cerebral. De esta manera, las técnicas de neuroimagen han comenzado a arrojar cierta luz sobre los cambios funcionales y estructurales que se producen en pacientes con daño cerebral cuando se interviene para recuperar la función dañada, es decir, sabemos qué ocurre en el cerebro antes y después de nuestra intervención, lo que a su vez permite comprender los efectos de la plasticidad cerebral.

En este sentido, los diferentes modelos psicoterapéuticos deben explicar algo más de lo que resulta obvio, es decir, deben ir más allá de la afirmación de que algo es eficaz, para explicar cómo y por qué es eficaz. Podíamos afirmar que cualquier intervención persigue someter al sujeto a una serie de experiencias (reales o imaginadas, pasadas, presentes o futuras) para que las relaciones cerebro-mente se orienten en una dirección más adecuada o adaptativa. Cuando hablamos de las relaciones cerebro-mente partimos de una premisa que ya hemos señalado anteriormente: cualquier proceso mental se sustenta en el funcionamiento cerebral aunque no puede ser explicado sólo por el resultado de dicho funcionamiento (reduccionismo codicioso en terminología de Dennett) ${ }^{(22)}$. Desde este planteamiento y sabiendo lo que sabemos sobre el funcionamiento cerebral la pregunta que debemos hacernos es la siguiente: ¿podemos explicar la eficacia de las técnicas basándonos en los modelos de dicho funcionamiento cerebral, sea normal o patológico? Nos tememos que esta pregunta pone en apuros a algunos tipos de intervención que utilizamos con frecuencia en nuestras consultas (o tal vez a los aplicadores de la técnica de intervención).

Para plantear esta relación entre modelos de funcionamiento cerebral e intervención psicoterapéutica hemos optado por el modelo jerárquico de Stuss y Benson por un doble motivo: por un lado es un modelo integrador que trata de plantear una jerarquía entre las diferentes funciones mentales y la estructura cerebral que las sustenta y por otro lado, es un modelo de obligada referencia en cualquier trabajo relacionado con una visión integradora sobre el funcionamiento cerebral.

Aunque debemos reconocer el carácter especulativo de este artículo, no resulta menos cierto que hoy en día nos hallamos inmersos en la vorágine de los datos y de los estudios de casos pero en ocasiones nos encontramos con pocos modelos que intenten integrar los datos existentes en un modelo comprensivo. En este sentido, este trabajo trata de comprender el efecto de la psicoterapia de las adicciones sobre los procesos mentales y sus correlatos en el funcionamiento cerebral. 
Como plantea Mora(23), la neurociencia actual está proporcionando un nuevo modo de entender el cerebro en su intento de unificar estos dos mundos (mente y cerebro). Hoy ya no consideramos el cerebro como un ente estático, sino que sabemos que se trata de un órgano en cambio permanente, tanto en su estructura como en su función. Se trata de un órgano neuronal plástico, lo que significa que el procesamiento neuronal produce un cambio constante en el propio procesador.

Se han repasado una serie de intervenciones terapéuticas que tratan de conseguir un cambio en la conducta. Este cambio será estable en la medida en que la nueva conducta muestre capacidad de modificar las conexiones entre neuronas. En definitiva, siguiendo a Kandel $^{(4)}$, nos hemos intentado acercar (de forma evidentemente especulativa) a conocer qué ocurre en la tarea de la psicoterapia, qué ocurre cuando un terapeuta habla a un paciente y éste escucha, qué cambios produce la maquinaria cerebral del terapeuta en la maquinaria neuronal del paciente.

\section{BIBLIOGRAFÍA}

1. Sanjuán J. Orígenes y fundamentos de los enfoques evolucionistas de la mente. En Sanjuán J (ed). Evolución cerebral y Psicopatología. Madrid: Triacastela; 2000.

2. Siegler M, Osmond H, Newell S. Models of alcoholism. En Freed EX. (ed), Interfaces between alcoholism and mental health. Rutgers Center of alcohol studies, New Brunswick, New Jersey. 1982.

3. Tirapu J, Lorea I, Landa N. Conductas adictivas: de la neurobiología al evolucionismo. Adicciones 2003; 3: 255-271.

4. Kandel E. A new intelectual framework for psychiatry. Am J Psychiatry 1998; 155: 457-469.

5. Tejero A, Pérez de los Cobos J, Bosch R, Siñol N. Adicciones, lóbulo frontal y funciones ejecutivas: un análisis neuropsicológico del autocontrol en las Adicciones. Conductas adictivas 2003;3 (1): 43-44.

6. Miller WR, Rollnick, S. Motivational interviewing: preparing people to change adictive behavior. New York: Guildford Press, 1991.

7. Carroll KM, Lobby B, Sheehan J, Hyland N. Motivational interviewing to enhance treatment initiation in substance abusers: an effectiveness study. Am J Addict $2001 ; 10: 335-9$.

8. Swanson AJ, Pantalon MV, Cohen KR. Motivational interviewing and treatment adherence among psychiatric and dually diagnosed patients. J Nerv Ment Dis 1999; 187:630-5.

9. Stotss AL, Schimitz JM, Rhoades HM, Grabowski J. Motivational interviewing with cocaine-dependent patients: a pilot study. J Consult Clin Psychol 2001; 69:858-62

10. Marlatt GA, Gordon, JR. (eds.), Relapse prevention. Maintenance strategies in the treatment of addictive behaviors. New York: The Guilfod Press; 1985.

11. Pineda J, Torrecilla M. Mecanismos neurobiológicos de la adicción a drogas. Trastornos Adictivos 1999; 1(1):1321.

12. Navarro J. Intervención en redes sociales de adictos. Revista de psicoterapia 1994;5:99-117.

13. Llitrà y Virgili E. Aproximación al concepto de apoyo familiar como variable en el tratamiento de las drogodependencias. Rev Esp Drog 1999; 24:336-368.

14. Broome KM, Simpson DD, Joe GW. The role of social support following short-term inpatient treatment. Am J Addict 2002; 11:57-65

15. Dobkin PL, De CM, Paraherakis A, Gill K. The role of funcional social support in treatment retention and outcomes among outpatient adult substance abusers. Addiction 2002; 97:347-356.

16. Wasserman DA, Sewart AL, Delucchi KL. Social support and abstinence from opiates and cocaine during opioid maintenance treatment. Drug Alcohol Depend 2001; 65:65-75.

17. Stuss DT, Benson DF: The frontal lobes. New York. Raven Press. 1986.

18. Stuss D.T:: Disturbance of self-awareness after frontal system damage. En: Prigatano G., Schacter D: Awareness of deficit after brain injury. New York. Oxford University Press. 1991.

19. Tirapu J, Muñoz-Céspedes JM, Pelegrín C. Funciones ejecutivas: necesidad de una integración conceptual. Rev Neurol 2002; 34(7): 673-685.

20. Volkow ND, Fowler JS, Wang GJ. The addicted human brain: insights from imaging studies. The Journal of Clinical investigation 2003; 111(10): 1444-1451.

21. Maggos CE, Tsukada H, Kakiuchi T, Nishiyama S, Myers JE, Kreuter J, Schlussman SD, Unterwald EM, Ho A, Kreek MJ. Sustained withdrawal allows normalization of in vivo $[11 \mathrm{C}] \mathrm{N}$-methylspiperone dopamine D2 receptor binding after chronic binge cocaine: a positron emission tomography study in rats. Neuropsychopharmacology 1998; 19(2):146-53.

22. Dennet D: La peligrosa idea de Darwin. Madrid. Galaxia Gutenberg.1999.

23. Mora F. El reloj de la sabiduría. Tiempos y espacios en el cerebro humano. Madrid: Alianza; 2001. 
